\title{
Maternal and Child Health (MCH) Handbook and Its Effect on Mater- nal and Child Health Care: A Systematic Review and Meta-Analysis
}

\author{
Shafi Bhuiyan ${ }^{1,2 *}$, Housne Begum ${ }^{2,3}$, Deena ${ }^{2}$, Sabeen Ehsan², Syed Jamal Shah ${ }^{2}$, Rabia Shariff ${ }^{2}$, Vanessa Linton ${ }^{2,3}$, Nafisa T Bhui- \\ yan $^{1}$
}

${ }^{1}$ Division of Clinical Public Health Dalla Lana School of Public Health University of Toronto, Canada

${ }^{2}$ Ryerson University, Toronto, Ontario, Canada

${ }^{3}$ McMaster University, Hamilton, Ontario, Canada

"Corresponding author: Shafi Bhuiyan, Division of Clinical Public Health Dalla Lana School of Public Health University of Toronto, Canada. Tel: +16477719299 Email: shafi.bhuiyan@utoronto.ca

Citation: Bhuiyan S, Begum H, Deena, Ehsan S, Jamal Shah S, et al. (2017) Maternal and Child Health (MCH) Handbook and Its Effect on Maternal and Child Health Care: A Systematic Review and Meta-Analysis. J Community Med Public Health: CMPH-109. DOI: 10.29011/CMPH-109/100009

Received Date: 02 August, 2017; Accepted Date: 25 August, 2017; Published Date: 02 September, 2017

\begin{abstract}
To search the literature for evidence for examining the effect of MCH Handbooks to promote and improve health outcomes of the Maternal and Child Health care in developing countries.

Pub Med, EMBASE, Cochrane, Web of Science, and Google Scholar were searched. Study quality and the risk of bias were evaluated using the Cochrane Handbook. A random effects meta-analysis was performed. The qualitative findings were also presented in a tabular form.

The search resulted in 359 studies and 30 articles were included for full text screening and only seven were included in the meta-analysis. The estimated Risk Ratio (RR) for knowledge, practice and attitude of mothers on Maternal and Child Health Care were better among $\mathrm{MCH}$ Handbook users than non- $\mathrm{MCH}$ Handbook users. When comparing non-MCH handbook users to $\mathrm{MCH}$ handbook users for women's knowledge of antenatal care visits, RR was 0.81 (95\% Confidence Interval [CI] 0.78-0.84) and for knowledge of danger signs RR was $0.51,95 \%$ CI $0.45-0.59$. Practice-related variables such as birth weight measured within $48 \mathrm{hrs}$ found RR $0.81,95 \%$ CI $0.79-0.82$. For delivery at health facility the RR when comparing non-MCH handbook users to $\mathrm{MCH}$ handbook users was $0.82,95 \%$ CI $0.62-1.08$ Finally, attitude-related variables such as positive changes in attitude on pregnancy care calculated RR $0.33,95 \%$ CI 0.14-0.81 when comparing non-MCH handbook users to MCH handbook users.

The positive impacts of the $\mathrm{MCH}$ Handbook on knowledge, practice, and attitude-related variables suggest that the $\mathrm{MCH}$ Handbook is an effective tool to promote the maternal and child health care. In addition, MCH Handbook may offer an alternative tool for educating mothers for better maternal and child health care. There is a need for additional research to explore gaps identified in the current literature.
\end{abstract}

Keywords: MCH Handbook; Maternal and Child Health; Utilization of Health Services

\section{Introduction}

Improving maternal and child health has been highlighted as a key public health concern since the year 2000, with the development of the Millennium Development Goals (MDGs). Compared to the other six MDGs, goal 4 for children's health and goal
5 for women's health continue to lag behind. To facilitate progress toward achieving these two goals, the global health community now pays special attention to Maternal, Neonates, and Child Health (MNCH) [1-5]. Larger and more effective interventions and investment in $\mathrm{MNCH}$ are necessary to achieve these healthrelated MDGs [6]. Providing quality care during pregnancy and child delivery remain a major challenge [7]. To fill these gaps, both demand- and supply-side interventions are necessary [8]. In this 


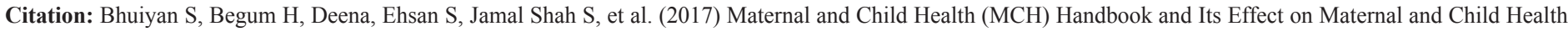
Care: A Systematic Review and Meta-Analysis. J Community Med Public Health: CMPH-109.

context, several countries adopted the Maternal and Child Health Handbook (the MCH Handbook) as a tool to promote better knowledge and service-seeking behavior among women [9].

The World Health Organization (WHO) has recommended the use of home-based records as a viable tool for ensuring the continuity of care for mothers and children before and after pregnancy [10]. Specifically, the WHO has identified some key recording tools such child health records, child immunization cards and counseling cards for childcare [10]. The MCH Handbook is a home-based health record for both the mother and child. It records the health condition of the mother throughout pregnancy, delivery, and the postnatal period, as well as the condition of the child before, at, and after birth, including immunization records and growth monitoring. It also contains health education information related to $\mathrm{MNCH}$. The $\mathrm{MCH}$ Handbook can be used to monitor the health of a woman and her child, keep record of the utilization of health services, promote health education, and provide information when either mother or child is referred. The MCH Handbook may empower women by facilitating greater participation in their own medical care [11].

The objective of this review was to examine the effect of $\mathrm{MCH}$ Handbooks on the promotion of maternal and child health in developing countries.

\section{Methods}

\section{Summary of Methods}

A systematic literature review and meta-analysis was carried out to examine the effect of the $\mathrm{MCH}$ Handbook on maternal and child health care. The focus was on different variables related to maternal health and child health such as changes in mothers' knowledge, practice and attitude. This review was conducted using the methods outlined in the Cochrane Handbook for Systematic Reviews of Interventions [12] and is reported according to the PRISMA Checklist.

\section{Literature Search}

A literature search was carried out for articles published in Medline, Pub Med, the Cochrane Library and Google Scholar. The literature search included the following search terms and keywords: "MCH Handbook" OR "Maternal and child health handbook" OR "Home-based record" OR "Paper-based record" OR "personal health record" OR "Child health record/book" OR "maternal health record/book" OR "Maternal and child health record/ book" OR "Vaccination record/card". The search term contained both controlled word and free text.

In addition, references were manually identified from the reference lists of key papers found during the searches and a few studies were manually identified as published online but not yet listed in literature databases. The search was not restricted to stud- ies published in English - although only those with translations to English were included. In order to be included, studies had to identify and measure effects of $\mathrm{MCH}$ Handbook on maternal and child health. The included analyses primarily used a meta-analysis of different variables related to maternal and child health in pre and post $\mathrm{MCH}$ Handbook situations. Narrative results were also presented if relevant in a separate table. Full papers were obtained and formally assessed for all studies that appeared to be potentially relevant. In addition, available abstracts related to effectiveness of $\mathrm{MCH}$ Handbook were also considered if relevant and sufficient for presentations in this review, acknowledging the limitation of this inclusion.

Ovid MEDLINE(R) In-Process \& Other Non-Indexed Citations, Ovid MEDLINE(R) Daily and Ovid MEDLINE(R) (1946 to April 16 2017), Embassy (1974 to April 16 2017) were systematically searched and also were searched in Google Scholar, Cochrane data base and were reviewed the 350 hits for potentially eligible studies (see PRISMA). Moreover, 10 articles were identified from additional sources.

\section{Selection of studies}

All identified articles were merged into a common file, and duplicates were deleted. Results were divided among two reviewers who independently examined the assigned articles and classified each as "Exclude", "Include", or "Unsure." A third reviewer settled discrepancies. Initial screening began with a title screen. Articles needed to include the words "MCH Handbook", "Maternal and Child Health Care". Next, abstracts were retrieved and screened to determine eligibility. Finally, full-text articles were retrieved and screened for inclusion.

\section{Eligibility Criteria}

\section{Inclusion criteria}

For title and abstract screening process, first we looked for existing Systematic Reviews (SR) on the MCH Handbook and its effectiveness Inclusion criteria for Title and Abstract Screening

Study type: We excluded reviews that were clearly narrative reviews or overviews of a topic that do not include reporting and synthesis of results of trials. We included relevant conference abstracts (and checked for follow-up publications), as far as they described to be a SR or original studies. We were looking for any primary study identified to conduct this systematic review.

Population: Studies including mothers using $\mathrm{MCH}$ Handbook and not using $\mathrm{MCH}$ Handbook (control). Intervention and comparison related: Intervention and comparison were mothers using the MCH Handbook and mothers not using the MCH Handbook. Interventions that were not relevant were excluded at the full-text screening stage.

Reported information (outcomes): The articles reporting mater- 


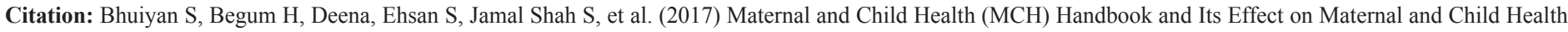
Care: A Systematic Review and Meta-Analysis. J Community Med Public Health: CMPH-109.

nal and child health-related variables in relation to $\mathrm{MCH}$ Handbook's effect were included. Variables included knowledge of mother on antenatal care visits, danger signs, breast feeding, and vaccination. Practice-related variables were practice of antenatal care visits/continue of care, birth weight measured within $48 \mathrm{hrs}$, delivery at health facility, trained attendant at birth, mother's tetanus taxied, breast feeding, child vaccination, vitamin A and iron supplementation. Finally, attitude-related variables included positive changes in attitude on pregnancy care, support of health staff during pregnancy, child care, and the role of their husband during the pregnancy period.

\section{Exclusion criteria}

Non-original studies, structured abstracts, project records, letters/commentary, case reports, and case series were excluded.

Duplicates: When we came across duplicate citations, moved into the specific folder.

\section{Full text screening}

The first step was title and abstract screening to identify studies appearing to meet the inclusion criteria, potentially relevant, or with sufficient information to make a clear judgment to be included. The second step was screening those studies after retrieving the full texts.

\section{Data extraction and management}

The included full text articles were randomly shuffled using Endnote X6 and then the articles were assigned to each reviewer for data extraction. A third reviewer handled dissension. Studies meeting the inclusion criteria were included for data extraction. A standardized data extraction form was developed, which was pilot tested on two full-text articles. Each team member independently reviewed the full-text article and the following details were Extracted: basic characteristics including first author, publication year; study population (type of population either mother using MCH Handbook or not, age), setting, country, interventions, outcomes (knowledge, practice and attitude related to maternal and child health/care), and additional comments (if any).

\section{Data Analysis (Quantitative and Narrative synthesis)}

Two investigators independently collected data for patient characteristics, diagnosis, treatments, setting, follow-up, and outcomes using a pretested data abstraction form. The quality/risk of bias was assessed for each outcome from the studies using the Cochrane risk of bias tool for RCTs [13]. Data were analyzed by using RevMan 5.2 (The Nordic Cochrane Center, Copenhagen, Denmark). Relative risks (e.g. Risk Ratios [RRs]) were calculated by pooling results from RCTs and non-RCTs comparing MCH Handbook and not MCH Handbook. Also, a narrative summary of the included studies with narrative findings were presented in a Table 1 with all other study characteristics such as basic study information characteristics- first author, publication year; study population (type of population either mother using $\mathrm{MCH}$ Handbook or not, age), setting, country, interventions, findings as a result of $\mathrm{MCH}$ Handbook utilization and additional comments (if any).

\section{Assessment of methodological quality of included studies}

Two investigators evaluated the certainty of the evidence for each outcome using the Grading of Recommendations, Assessment, Development and Evaluation (GRADE) approach, and resolved any discrepancies [13]. The following GRADE domains were assessed: risk of bias, inconsistency, imprecision, indirectness, publication bias, magnitude of effect, and opposing plausible confounding [13].

\section{Results}

\section{Search results}

Among 359 non-duplicate records identified from the electronic database search and from other sources, 30 articles in full text were retrieved after title and abstract screening (Figure 1). After exclusion of articles that were not relevant, 14studies were included. Seven articles were found for the quantitative analysis, and seven articles were found for the narrative summary. Only one article was an RCT and the rest were nonrandomized studies comparing effect of MCH Handbooks to non- MCH Handbooks or pre and post-MCH Handbook situations. Figure -1 
Citation: Bhuiyan S, Begum H, Deena, Ehsan S, Jamal Shah S, et al. (2017) Maternal and Child Health (MCH) Handbook and Its Effect on Maternal and Child Health Care: A Systematic Review and Meta-Analysis. J Community Med Public Health: CMPH-109.

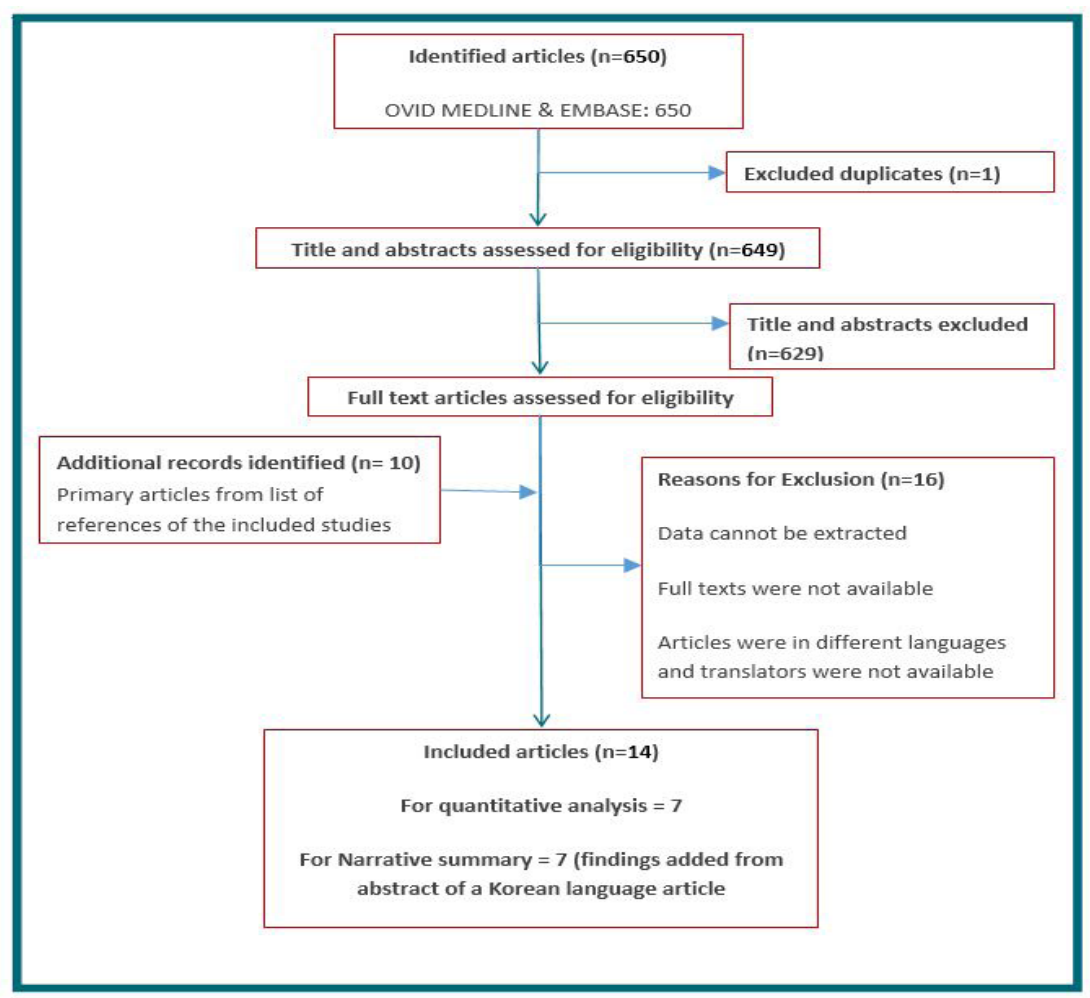

Figure 1: PRISMA.

\section{Comparisons of effect of MCH Handbook and Non-MCH Handbook}

Only one RCT and six non-RCTs were identified for comparing the effect of MCH Handbook and non-MCH Handbook. These seven studies compared the effects of MCH Handbook and non-MCH Handbook on maternal knowledge, practice and attitude on MCH health care. [14-20]. when direct comparisons within studies were available, relative risks and risk differences were calculated (Figures $2-4)$ and also variables measured related to knowledge, practice and attitude were shown in the same figures. When comparing women's knowledge of antenatal care visits between non- $\mathrm{MCH}$ Handbook and $\mathrm{MCH}$ handbook scenarios, the RR was 0.81 (95\% CI 0.78-0.84).

Similarly, when comparing non-MCH Handbook Users to $\mathrm{MCH}$ handbook users, $\mathrm{MCH}$ handbook users had lower knowledge of a range of topics including danger signs (RR 0.51; 95\% confidence interval [CI] 0.45-0.59), breast feeding (RR 0.73; 95\% CI 0.69-0.78), and vaccination (RR $0.18 ; 95 \%$ CI 0.11-0.28). In situations where the MCH Handbook was not used, practice-related events were less likely to occur such as practice of antenatal care visits/continue of care (RR $0.76 ; 95 \%$ CI $0.67-0.87$ ), birth weight measured within $48 \mathrm{hrs}$ (RR $0.81 ; 95 \%$ CI $0.79-0.82$ ), delivery at health facility (RR 0.82 ; 95\% CI $0.62-1.08$ ), trained attendant at birth (RR $0.85 ; 95 \%$ CI 0.78 0.93), mother's tetanus taxied(RR 0.47; 95\% CI 0.42-0.53), breast feeding (RR 0.24; 95\% CI 0.03-1.68), child vaccination (RR 0.37; 95\% CI 0.25-0.57), vitamin A and iron supplementation (RR 0.08; 95\% CI 0.03-0.20).

Finally, studies examined the impact of the MCH handbook use compared to situations where the MCH Handbook was not used on attitude-related variables. It was found that non- $\mathrm{MCH}$ Handbook users were less likely to experience positive attitude-related variables such as positive changes in attitude on pregnancy care (RR 0.33; 95\% CI 0.14-0.81), support of health staff during pregnancy (RR $0.58 ; 95 \%$ CI $0.32-1.05$ ), child care (RR $0.43 ; 95 \%$ CI $0.21-0.90$ ), and the role of their husband during the pregnancy period (RR 0.89 ; 95\% CI 0.38-0.2.08) Detailed results are shown in Figures 2-4. The study characteristics of all these seven studies are also presented in (Table 1). 


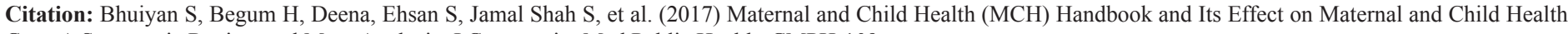
Care: A Systematic Review and Meta-Analysis. J Community Med Public Health: CMPH-109.

\begin{tabular}{|c|c|c|c|c|c|c|c|c|}
\hline \multicolumn{9}{|c|}{ Included study characteristics Table 1} \\
\hline $\begin{array}{l}\text { Study } \\
\text { Year } \\
\text { Country }\end{array}$ & $\begin{array}{l}\text { Type of } \\
\text { Study De- } \\
\text { sign }\end{array}$ & Population & $\begin{array}{l}\text { Age Mean } \\
\text { (Sd), } \\
\text { Range }\end{array}$ & $\begin{array}{l}\text { No. Of Par- } \\
\text { ticipants }\end{array}$ & Interventions & $\begin{array}{l}\text { Inclusion } \\
\text { Criteria }\end{array}$ & $\begin{array}{l}\text { Exclusion } \\
\text { Criteria }\end{array}$ & Outcomes \\
\hline $\begin{array}{c}\text { Aiga } 2016 \\
\text { Vietnam [14] }\end{array}$ & $\begin{array}{l}\text { Comparison } \\
\text { of pre \& } \\
\text { post inter- } \\
\text { vention }\end{array}$ & $\begin{array}{c}\text { Pregnant } \\
\text { women/ } \\
\text { mothers of } \\
\text { children 6-18 } \\
\text { months of age }\end{array}$ & $15->34$ & $\begin{array}{c}\text { MCHHB } \\
(\mathrm{n}=810 . .) \\
\text { NonMCHHB } \\
(\mathrm{n}=810)\end{array}$ & $\begin{array}{l}\text { MCHHB Vs. } \\
\text { No MCHHB }\end{array}$ & $\begin{array}{l}\text { Women from } \\
\text { four specific } \\
\text { provinces } \\
\text { (selected as } \\
\text { pilot prov- } \\
\text { inces) were } \\
\text { randomly } \\
\text { selected }\end{array}$ & $\begin{array}{l}\text { Not men- } \\
\text { tioned }\end{array}$ & $\begin{array}{c}\text { Practice: antenatal } \\
\text { care visits, promo- } \\
\text { tion of ANC atten- } \\
\text { dance, delivery with } \\
\text { SBAs, delivery at a } \\
\text { health facility } \\
\text { Knowledge: antena- } \\
\text { tal care visits, danger } \\
\text { signs } \\
\text { breast feeding } \\
\text { Attitude: on support } \\
\text { of health staff during } \\
\text { pregnancy }\end{array}$ \\
\hline $\begin{array}{l}\text { Bhuiyan } 2006 \\
\text { Bangladesh[15] }\end{array}$ & $\begin{array}{l}\text { Case Con- } \\
\text { trol study } \\
\text { using pre \& } \\
\text { post inter- } \\
\text { vention }\end{array}$ & $\begin{array}{l}\text { Pregnant } \\
\text { women } \\
\text { visiting } \\
\text { Maternal and } \\
\text { Child Health } \\
\text { Training } \\
\text { Institute first } \\
\text { time during } \\
\text { the current } \\
\text { pregnancy }\end{array}$ & $>20$ & $\begin{array}{c}\text { Case (with) } \\
\text { MCHHB } \\
(\mathrm{n}=240) \\
\text { Control (with- } \\
\text { out MCHHB) } \\
(\mathrm{n}=360)\end{array}$ & $\begin{array}{l}\text { Use (introduc- } \\
\text { tion) of } \mathrm{MCH} \\
\text { booklet vs. } \\
\text { traditional } \\
\text { health cards }\end{array}$ & $\begin{array}{l}\text { Pregnant } \\
\text { women } \\
\text { visiting } \\
\text { Maternal and } \\
\text { Child Health } \\
\text { Training } \\
\text { Institute first } \\
\text { time during } \\
\text { the current } \\
\text { pregnancy }\end{array}$ & $\begin{array}{l}\text { Not men- } \\
\text { tioned }\end{array}$ & $\begin{array}{c}\text { Practice: antenatal } \\
\text { care visits, promo- } \\
\text { tion of ANC atten- } \\
\text { dance, delivery with } \\
\text { SBAs, delivery at a } \\
\text { health facility } \\
\text { Knowledge: antena- } \\
\text { tal care visits, danger } \\
\text { signs } \\
\text { breast feeding, child } \\
\text { vaccination, vitamin } \\
\text { A and iron supple- } \\
\text { mentation, Family } \\
\text { planning } \\
\text { Attitude: positive } \\
\text { attitude on preg- } \\
\text { nancy care, support } \\
\text { of health staff during } \\
\text { pregnancy, child care }\end{array}$ \\
\hline $\begin{array}{l}\text { Kawakatsu } \\
2015 \\
\text { Kenya [16] }\end{array}$ & $\begin{array}{l}\text { A commu- } \\
\text { nity-based } \\
\text { cross- } \\
\text { sectional } \\
\text { survey }\end{array}$ & $\begin{array}{l}\text { Mothers who } \\
\text { had children } \\
\text { aged 12-23 } \\
\text { months }\end{array}$ & $>20$ & $\begin{array}{c}\text { Treatment } \\
(\mathrm{N}=1331) \\
\text { Control } \\
(\mathrm{N}=652)\end{array}$ & $\begin{array}{l}\text { Treatment } \\
\text { ('Possess an } \\
\text { MCH Hand- } \\
\text { book' } \\
\text { Control (or } \\
\text { 'Lost or } \\
\text { never owned a } \\
\text { Handbook' }\end{array}$ & $\begin{array}{l}\text { The study } \\
\text { population } \\
\text { comprised all } \\
\text { mothers in } \\
\text { the research } \\
\text { area who had } \\
\text { children aged } \\
12-23 \text { months }\end{array}$ & $\begin{array}{l}\text { Not men- } \\
\text { tioned }\end{array}$ & $\begin{array}{l}\text { Practice: antenatal } \\
\text { care visits } \\
\text { Knowledge: antena- } \\
\text { tal care visits } \\
\text { Practice: delivery at } \\
\text { health facility }\end{array}$ \\
\hline \multicolumn{9}{|c|}{ Included study characteristics (continue of Table 1) } \\
\hline $\begin{array}{l}\text { Study } \\
\text { Year } \\
\text { Country }\end{array}$ & $\begin{array}{l}\text { Type of } \\
\text { Study De- } \\
\text { sign }\end{array}$ & Population & $\begin{array}{l}\text { Age Mean } \\
\text { (Sd), } \\
\text { Range }\end{array}$ & $\begin{array}{l}\text { No. Of Par- } \\
\text { ticipants }\end{array}$ & Interventions & $\begin{array}{l}\text { Inclusion } \\
\text { Criteria }\end{array}$ & $\begin{array}{l}\text { Exclusion } \\
\text { Criteria }\end{array}$ & $\begin{array}{c}\text { Outcomes (No Need } \\
\text { To Include The } \\
\text { Numbers) }\end{array}$ \\
\hline
\end{tabular}




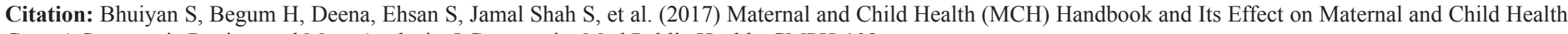
Care: A Systematic Review and Meta-Analysis. J Community Med Public Health: CMPH-109.

\begin{tabular}{|c|c|c|c|c|c|c|c|c|}
\hline $\begin{array}{c}\text { Mori } 2015 \\
\text { Mongolia [17] }\end{array}$ & $\begin{array}{c}\text { Cluster } \\
\text { Randomized } \\
\text { Controlled } \\
\text { Trial }\end{array}$ & $\begin{array}{l}\text { Pregnant } \\
\text { women and } \\
\text { their infants }\end{array}$ & $\begin{array}{l}\text { Interven- } \\
\text { tion group } \\
=27.3 \\
(6.13) \\
\text { Control } \\
\text { group= } \\
27.7(5.67)\end{array}$ & $\begin{array}{l}\text { Intervention } \\
\text { group- } 253 \\
\text { women and } \\
\text { control group } \\
248 \text { women }\end{array}$ & $\begin{array}{l}\text { MCHH group, } \\
\text { control group }\end{array}$ & $\begin{array}{c}\text { Pregnant } \\
\text { women living } \\
\text { in the Bulgan } \\
\text { province of } \\
\text { Mongolia }\end{array}$ & $\begin{array}{l}\text { Not men- } \\
\text { tioned }\end{array}$ & $\begin{array}{l}\text { Practice: antenatal } \\
\text { care visits, healthy } \\
\text { behaviors such as } \\
\text { drinking water dur- } \\
\text { ing pregnancy, breast } \\
\text { feeding } \\
\text { Knowledge: antena- } \\
\text { tal care visits }\end{array}$ \\
\hline $\begin{array}{c}\text { Osaki } 2015 \\
\text { Indonesia [18] }\end{array}$ & $\begin{array}{l}\text { Cross-sec- } \\
\text { tional }\end{array}$ & $\begin{array}{l}\text { Respondents } \\
\text { with } 0 \text { to } \\
\text { 23-month-old } \\
\text { children }\end{array}$ & $\begin{array}{c}\text { Interven- } \\
\text { tion group } \\
28.89(6.2) \\
\text { Control } \\
\text { group } \\
29.54(6.8)\end{array}$ & $\begin{array}{c}\mathrm{MCHHB} \\
\mathrm{N}=4816 \\
\text { Single/no re- } \\
\text { cord } \mathrm{n}=3679\end{array}$ & $\begin{array}{l}\mathrm{MCHHB}, \\
\text { Single/no } \\
\text { record }\end{array}$ & $\begin{array}{c}\text { Mothers } \\
\text { with } 0 \text { to } \\
\text { 23-month-old } \\
\text { children }\end{array}$ & $\begin{array}{l}\text { Not men- } \\
\text { tioned }\end{array}$ & $\begin{array}{l}\text { Practice: antenatal } \\
\text { care visits, pro- } \\
\text { motion of ANC } \\
\text { attendance, delivery } \\
\text { with SBAs (skill } \\
\text { birth attendant), } \\
\text { birth weight mea- } \\
\text { sured within 48hrs, } \\
\text { delivery at a health } \\
\text { facility } \\
\text { Attitude: mother's } \\
\text { tetanus taxied, child } \\
\text { vaccination }\end{array}$ \\
\hline $\begin{array}{c}\text { Osaki } 2013 \\
\text { Indonesia [19] }\end{array}$ & $\begin{array}{l}\text { Cross-sec- } \\
\text { tional study }\end{array}$ & $\begin{array}{l}\text { Respondents } \\
\text { with } 0 \text { to } \\
\text { 23-month-old } \\
\text { children }\end{array}$ & $\begin{array}{c}\text { Interven- } \\
\text { tion group } \\
28.89(6.2) \\
\text { Control } \\
\text { group } \\
29.54(6.8)\end{array}$ & $\begin{array}{c}\text { MCHHB } \\
\mathrm{N}=301 \\
\text { Single/no } \\
\text { record } \mathrm{n}=96\end{array}$ & $\begin{array}{l}\text { MCHHB, } \\
\text { Single/no } \\
\text { record }\end{array}$ & $\begin{array}{c}\text { Mothers } \\
\text { with } 0 \text { to } \\
\text { 23-month-old } \\
\text { children }\end{array}$ & $\begin{array}{l}\text { Not men- } \\
\text { tioned }\end{array}$ & $\begin{array}{l}\text { Practice: antenatal } \\
\text { care visits, promo- } \\
\text { tion of ANC atten- } \\
\text { dance, delivery with } \\
\text { SBAs (skill birth } \\
\text { attendant), delivery } \\
\text { at a health facility } \\
\text { Attitude: mother's } \\
\text { tetanus taxied, child } \\
\text { vaccination }\end{array}$ \\
\hline $\begin{array}{c}\text { Yanagisawa } \\
2014 \\
\text { Cambodia [20] }\end{array}$ & $\begin{array}{l}\text { Case Con- } \\
\text { trol study } \\
\text { using pre \& } \\
\text { post inter- } \\
\text { vention }\end{array}$ & $\begin{array}{l}\text { Women who } \\
\text { have given } \\
\text { birth one year } \\
\text { earlier }\end{array}$ & $15-49$ & $\begin{array}{c}\text { MCHHB } \\
(\mathrm{n}=.320 .) \\
\text { NonMCHHB } \\
(\mathrm{n}=320)\end{array}$ & $\begin{array}{l}\text { Introduction } \\
\text { of MCHHB in } \\
\text { selected study } \\
\text { areas vs Non } \\
\text { MCHHB }\end{array}$ & $\begin{array}{l}\text { Living in the } \\
\text { intervention } \\
\text { and control } \\
\text { areas }\end{array}$ & $\begin{array}{l}\text { Not men- } \\
\text { tioned }\end{array}$ & $\begin{array}{c}\text { Practice: promotion } \\
\text { of ANC attendance, } \\
\text { delivery with SBAs, } \\
\text { delivery at a health } \\
\text { facility } \\
\text { Knowledge: danger } \\
\text { signs, breast feeding } \\
\text { Attitude: on support } \\
\text { of health staff during } \\
\text { pregnancy }\end{array}$ \\
\hline
\end{tabular}

Table 1: Study characteristics of included studies for meta-analysis

Eleven studies examined narrative findings on the same issues. [9,21-27] The available data suggested that there is positive effect of MCH Handbook on maternal and child health the quality of the evidence for almost all outcomes was low because there was only one RCT (with small sample size). The rest of the studies were non-randomized studies that compared the non-MCH Handbook with $\mathrm{MCH}$ Handbook and had low quality because of imprecise results due to few events and participants in the studies (Figure -2-4). 
Citation: Bhuiyan S, Begum H, Deena, Ehsan S, Jamal Shah S, et al. (2017) Maternal and Child Health (MCH) Handbook and Its Effect on Maternal and Child Health Care: A Systematic Review and Meta-Analysis. J Community Med Public Health: CMPH-109.

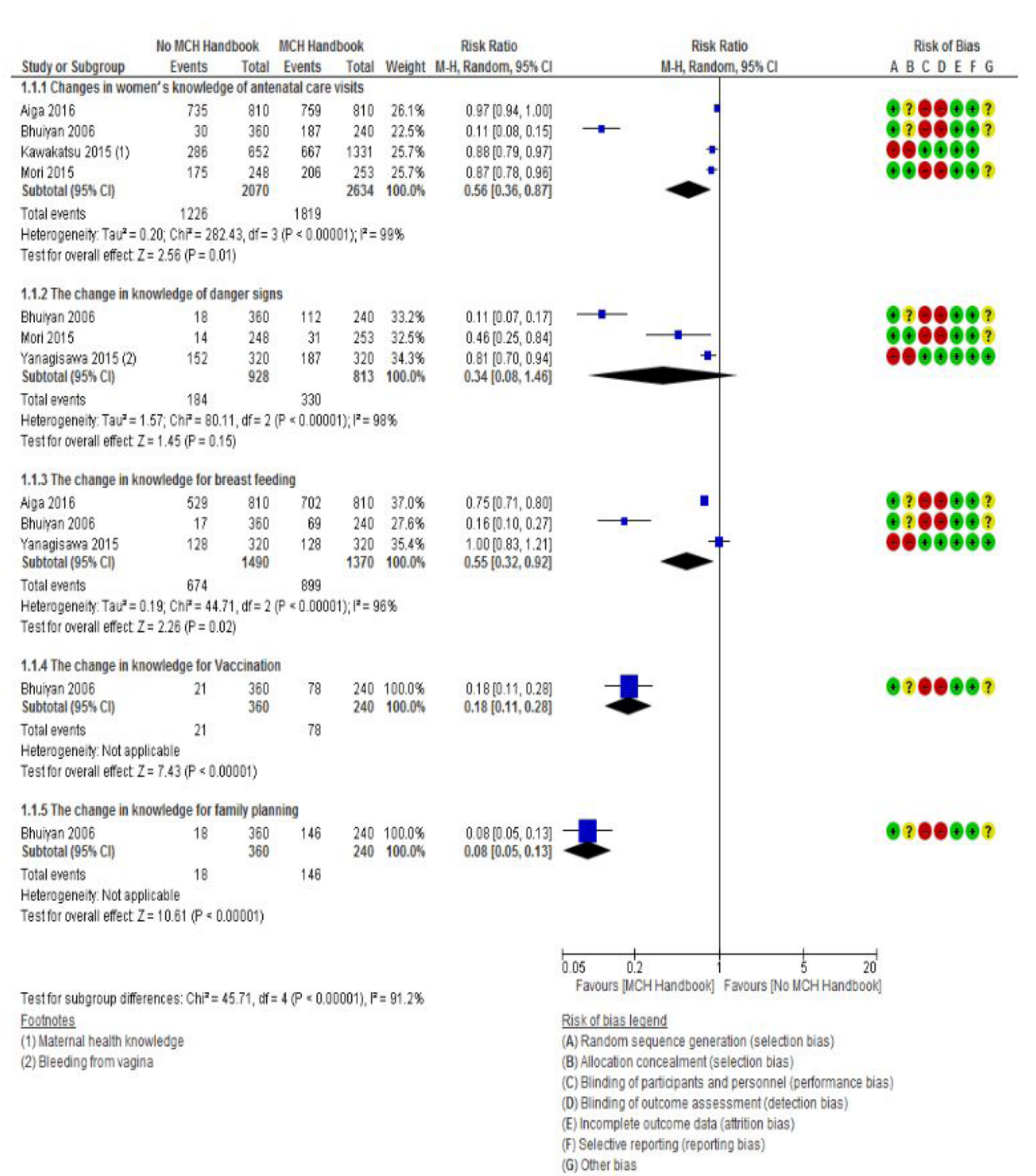

Figure 2: Comparison between MCH Handbook vs No MCH Handbook: Impact on Knowledge. 
Citation: Bhuiyan S, Begum H, Deena, Ehsan S, Jamal Shah S, et al. (2017) Maternal and Child Health (MCH) Handbook and Its Effect on Maternal and Child Health Care: A Systematic Review and Meta-Analysis. J Community Med Public Health: CMPH-109.

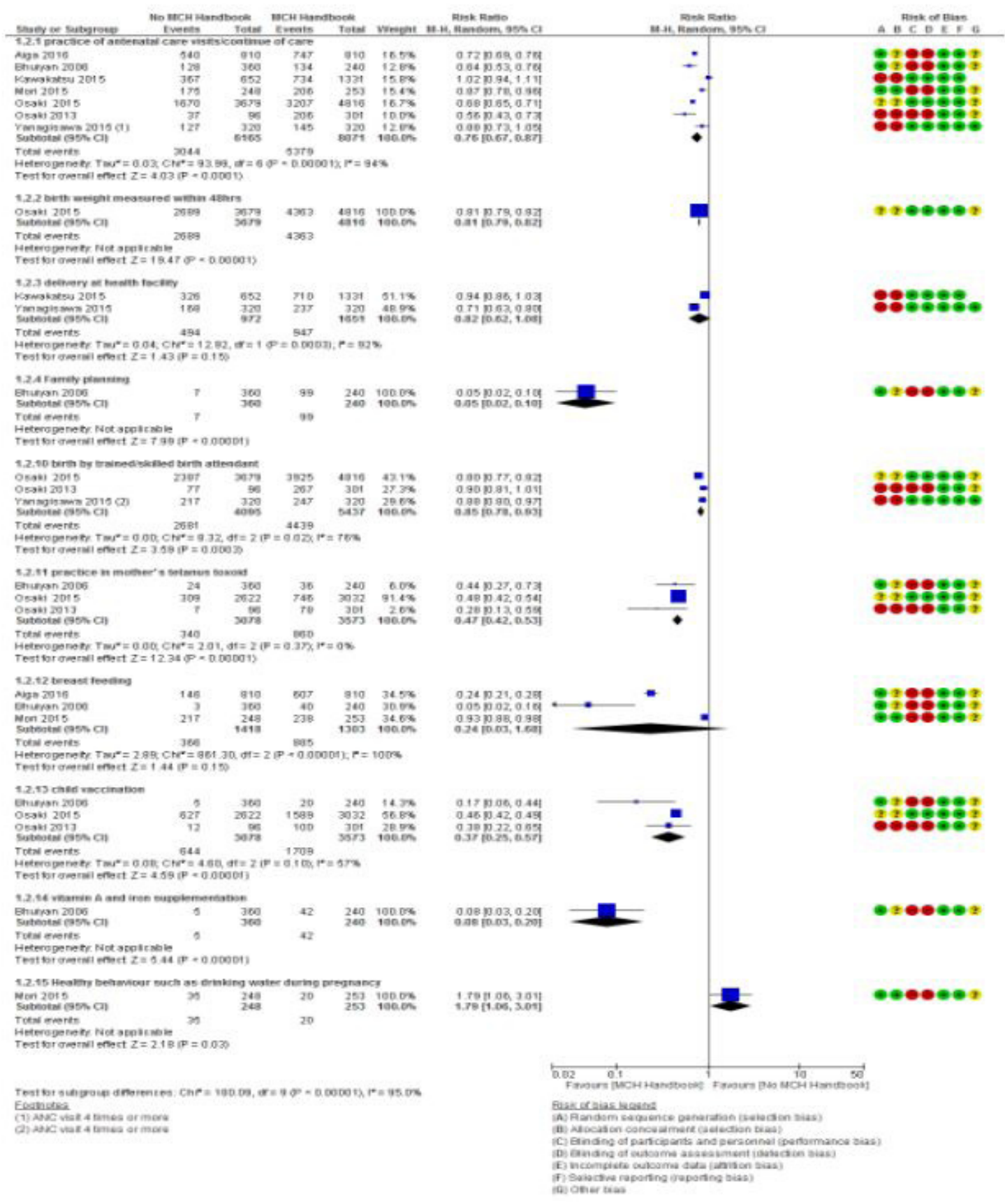

Figure 3: Comparison between MCH Handbook vs No MCH Handbook: Impact on Practice. 
Citation: Bhuiyan S, Begum H, Deena, Ehsan S, Jamal Shah S, et al. (2017) Maternal and Child Health (MCH) Handbook and Its Effect on Maternal and Child Health Care: A Systematic Review and Meta-Analysis. J Community Med Public Health: CMPH-109.

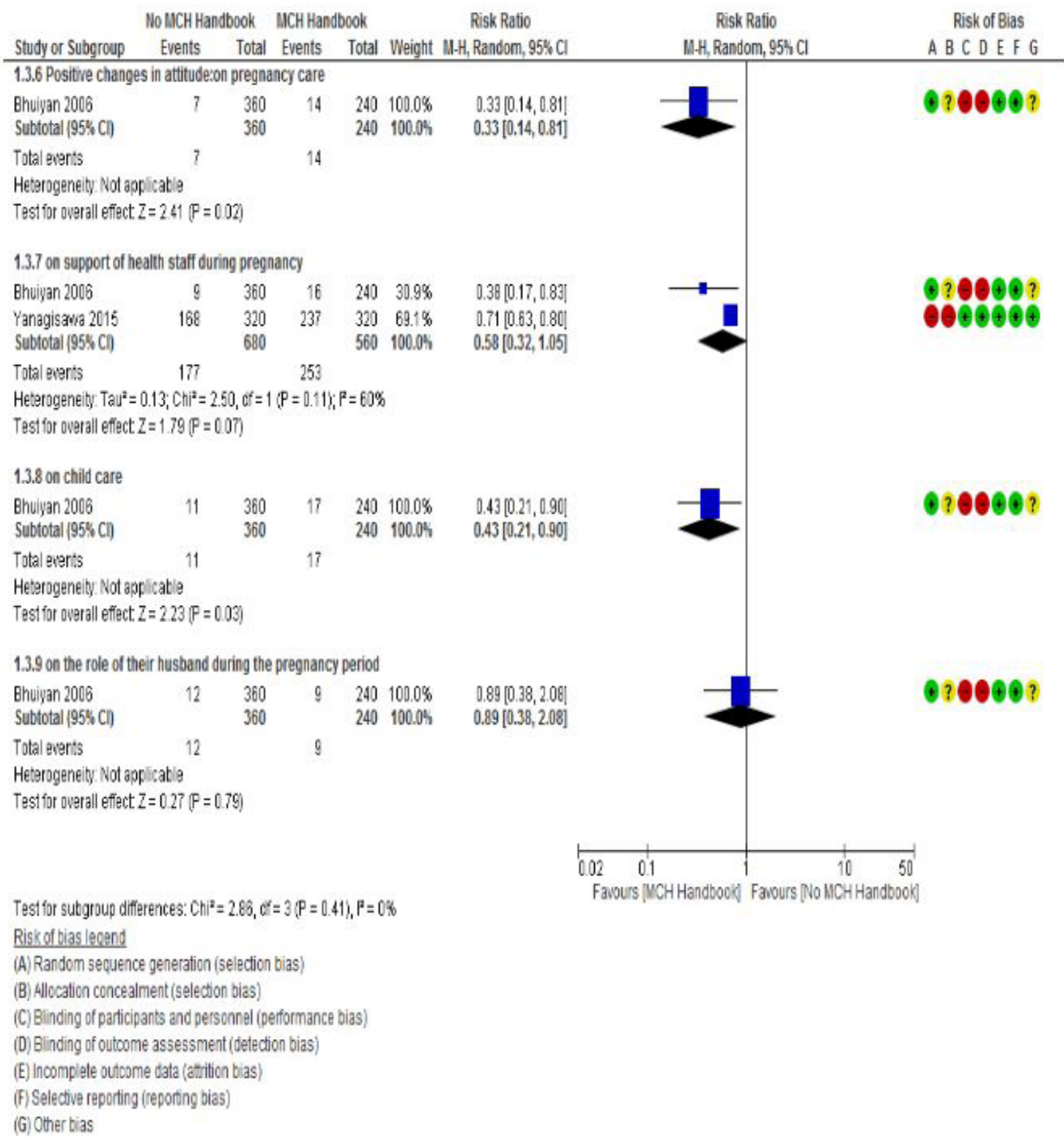

Figure 4: Comparison between MCH Handbook vs No MCH Handbook: Impact on Attitude. Table 2 


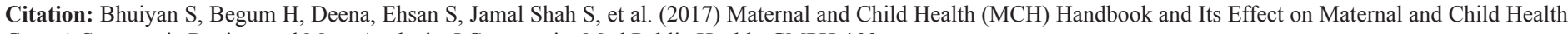
Care: A Systematic Review and Meta-Analysis. J Community Med Public Health: CMPH-109.

\begin{tabular}{|c|c|c|c|c|c|c|c|}
\hline $\begin{array}{l}\text { Study, Year, } \\
\text { Country }\end{array}$ & $\begin{array}{c}\text { Type of Study } \\
\text { Design }\end{array}$ & Population & $\begin{array}{c}\text { Age, Mean } \\
\text { Sd Range }\end{array}$ & $\begin{array}{c}\text { No. Of } \\
\text { Participants }\end{array}$ & Interventions & Inclusion Criteria & Findings \\
\hline $\begin{array}{c}\text { Bhuiyan } \\
2009 \\
\text { Bangladesh } \\
\text { [9] }\end{array}$ & $\begin{array}{c}\text { Cross } \\
\text { sectional } \\
\text { survey }\end{array}$ & Pregnant women & $\begin{array}{c}\text { Not } \\
\text { mentioned }\end{array}$ & 240 & $\begin{array}{c}\mathrm{MCH} \\
\text { Handbook }\end{array}$ & $\begin{array}{l}\text { pregnant women } \\
\text { of MCH Handbook } \\
\text { areas }\end{array}$ & $\begin{array}{l}\text { Improvement } \\
\text { in maternal } \\
\text { knowledge, attitude, } \\
\text { and utilization of } \\
\text { MCH services. In } \\
2007 \text { study, } 91 \% \\
\text { mothers could } \\
\text { read, understand, } \\
\text { make notes on the } \\
\text { MCH Handbook, } \\
\text { and also carried it } \\
\text { to consultations, } \\
\text { and only } 0.5 \% \\
\text { mothers lost their } \\
\text { handbooks. }\end{array}$ \\
\hline $\begin{array}{l}\text { Dagvadorj } \\
2017, \\
\text { Mongolia } \\
\text { [21] }\end{array}$ & $\begin{array}{c}\text { Longitudinal } \\
\text { Randomised } \\
\text { Control Trial } \\
\text { (RCT) 2010- } \\
2013\end{array}$ & $\begin{array}{l}\text { Mothers who } \\
\text { gave birth } \\
\text { and the three-year } \\
\text { follow-up if they } \\
\text { still lived in the } \\
\text { area. }\end{array}$ & $\begin{array}{c}\text { Not } \\
\text { mentioned }\end{array}$ & $\begin{array}{c}\text { Intervention } \\
\text { group } n= \\
214 \\
\text { control } \\
\text { group } n=172\end{array}$ & $\begin{array}{l}\text { MCHHB* Vs. } \\
\text { No MCHHB }\end{array}$ & $\begin{array}{l}\text { All women living in } \\
\text { the Bulgan province } \\
\text { of Mongolia who } \\
\text { gave birth between } \\
\text { March-August } 2010 \\
\text { participated in the } \\
\text { study }\end{array}$ & $\begin{array}{l}\text { Active usage of the } \\
\text { MCH Handbook } \\
\text { by the mothers for } \\
\text { three years helped } \\
\text { to lower the risk of } \\
\text { impaired cognitive } \\
\text { development }\end{array}$ \\
\hline $\begin{array}{l}\text { Fujimoto } \\
2001 \\
\text { Japan } \\
\text { [22] }\end{array}$ & $\begin{array}{c}\text { Questionnaire } \\
\text { survey }\end{array}$ & $\begin{array}{c}\text { Guardians } \\
\text { who visited } \\
\text { health stations } \\
\text { for } 18 \text {-month } \\
\text { examinations of } \\
\text { their children } \\
\text { and agreed to } \\
\text { participated in the } \\
\text { research }\end{array}$ & $\begin{array}{c}\text { Not } \\
\text { mentioned }\end{array}$ & $\begin{array}{c}10,900 \\
\text { guardians }\end{array}$ & МCHHB & $\begin{array}{l}13,271 \text { guardians } \\
\text { who visited health } \\
\text { stations for } 18- \\
\text { month examinations } \\
\text { of their children } \\
\text { and agreed to } \\
\text { participated in our } \\
\text { research }\end{array}$ & $\begin{array}{c}87.0 \% \text { of } \\
\text { respondents } \\
\text { answered that } \mathrm{MCH} \\
\text { Handbook was } \\
\text { helpful for child } \\
\text { bearing and } 81.6 \% \\
\text { said that the record } \\
\text { for immunization } \\
\text { was useful. } \\
\text { However, } 34.1 \% \\
\text { of respondents } \\
\text { answered it was not } \\
\text { simple to utilize } \\
\text { MCH Handbook } \\
\text { and } 60.6 \% \text { of them } \\
\text { requested more } \\
\text { detail on child } \\
\text { bearing. As for } \\
\text { dental health, the } \\
\text { completion rate for } \\
\text { information was } \\
\text { low and only } 21.3 \% \\
\text { of respondents } \\
\text { reported for the } \\
\text { dental record was } \\
\text { useful. }\end{array}$ \\
\hline
\end{tabular}




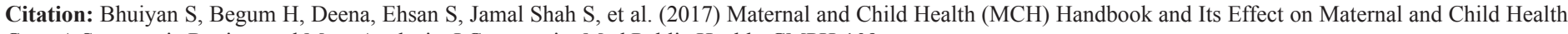
Care: A Systematic Review and Meta-Analysis. J Community Med Public Health: CMPH-109.

\begin{tabular}{|c|c|c|c|c|c|c|c|}
\hline $\begin{array}{l}\text { Study, Year, } \\
\text { Country }\end{array}$ & $\begin{array}{l}\text { Type of Study } \\
\text { Design }\end{array}$ & Population & $\begin{array}{l}\text { Age, Mean } \\
\text { Sd Range }\end{array}$ & $\begin{array}{c}\text { No. Of } \\
\text { Participants }\end{array}$ & Interventions & Inclusion Criteria & Findings \\
\hline $\begin{array}{c}\text { Hagiwaraa, } \\
2013 \\
\text { Palestine } \\
{[23]}\end{array}$ & $\begin{array}{l}\text { Case control } \\
\text { study }\end{array}$ & $\begin{array}{l}\text { Women coming } \\
\text { to } \mathrm{MCH} \text { treatment } \\
\text { centers }\end{array}$ & $\begin{array}{c}\text { Not } \\
\text { mentioned }\end{array}$ & $\begin{array}{c}\text { MCHHB } \\
n=270 ; \text { No } \\
\text { MCHHB } \\
n=70\end{array}$ & $\begin{array}{c}\text { MCH } \\
\text { Handbook vs } \\
\text { No MCHHB }\end{array}$ & $\begin{array}{l}\text { Women coming } \\
\text { to } \mathrm{MCH} \text { treatment } \\
\text { centers }\end{array}$ & $\begin{array}{l}\text { Knowledge related } \\
\text { on exclusive } \\
\text { breastfeeding and } \\
\text { how to cope with } \\
\text { the risks of rupture } \\
\text { of membranes } \\
\text { during pregnancy } \\
\text { increased among } \\
\text { MCH Handbook } \\
\text { users, especially } \\
\text { among less- } \\
\text { educated women. }\end{array}$ \\
\hline $\begin{array}{l}\text { Jeong } 2003 \\
\text { Korea [24] }\end{array}$ & $\begin{array}{l}\text { Cross- } \\
\text { sectional }\end{array}$ & $\begin{array}{l}\text { women whose } \\
\text { children were } \\
\text { between four and } \\
\text { six years old }\end{array}$ & $\begin{array}{c}\text { Not } \\
\text { mentioned }\end{array}$ & 312 & $\begin{array}{c}\mathrm{MCH} \\
\text { Handbook }\end{array}$ & $\begin{array}{l}\text { Women with } \\
\text { children between } \\
\text { four and six years } \\
\text { old, and residing } \\
\text { in six provinces of } \\
\text { Gyungsangnam, } \\
\text { Korea }\end{array}$ & $\begin{array}{l}\text { The awareness } \\
\text { and rate of DPT } \\
\text { (Diphtheria, } \\
\text { Pertussis, Tetanus } \\
\text { vaccine) additional } \\
\text { immunization was } \\
\text { significantly higher } \\
\text { in the women who } \\
\text { retained the MCH } \\
\text { Handbook than } \\
\text { their counterparts. }\end{array}$ \\
\hline $\begin{array}{c}\text { Kusumayati } \\
2007 \\
\text { Indonesia } \\
{[25]}\end{array}$ & $\begin{array}{l}\text { Cross } \\
\text { Sectional } \\
\text { Study }\end{array}$ & $\begin{array}{l}\text { mothers(pregnant } \\
\text { or with one or } \\
\text { more children } \\
\text { under age 3) }\end{array}$ & NA & $\begin{array}{c}\text { No MCHHB } \\
n=611 ; \\
\text { MCHHB } n= \\
630\end{array}$ & $\begin{array}{c}\mathrm{MCH} \\
\text { Handbook }\end{array}$ & $\begin{array}{l}\text { Mothers (pregnant } \\
\text { or with one or more } \\
\text { children under age } \\
\text { 3) }\end{array}$ & $\begin{array}{c}\text { Utilization of } \\
\text { MCHH has the } \\
\text { potential both to } \\
\text { improve maternal } \\
\text { knowledge and to } \\
\text { increase utilization } \\
\text { of maternal health } \\
\text { services }\end{array}$ \\
\hline $\begin{array}{c}\text { Osaki } 2009 \\
\text { Indonesia } \\
{[26]}\end{array}$ & $\begin{array}{l}\text { Retrospective } \\
\text { review }\end{array}$ & $\begin{array}{l}\text { Records of } \\
\text { Children 12-23 } \\
\text { months }\end{array}$ & $\begin{array}{c}12-23 \\
\text { months }\end{array}$ & $\begin{array}{c}\mathrm{n}=865 \\
(2002-3) \\
\text { and } \mathrm{n}=974 \\
(1997)\end{array}$ & $\begin{array}{c}\text { MCH } \\
\text { Handbook }\end{array}$ & $\begin{array}{l}\text { Children 12-23 } \\
\text { months }\end{array}$ & $\begin{array}{l}\text { Ownership of } \\
\text { home-based } \\
\text { immunization } \\
\text { records among } \\
\text { children aged 12-23 } \\
\text { months increased } \\
\text { from } 30.8 \% \text { (n } \\
=954) \text { in } 1997 \\
\text { and } 30.7 \%(\mathrm{n}= \\
865) \text { in } 2002-3 \\
\text { to } 37 \% \text { in } 2007 \text {. } \\
\text { This ownership } \\
\text { of immunization } \\
\text { record is associated } \\
\text { with greater } \\
\text { immunization } \\
\text { coverage }\end{array}$ \\
\hline
\end{tabular}

Table 2: Narrative summary of results from different studies. 


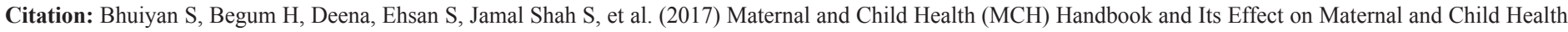
Care: A Systematic Review and Meta-Analysis. J Community Med Public Health: CMPH-109.

\section{Discussion}

The present systematic review of the literature was conducted to inform decision making about effect of the MCH Handbook on maternal and child care. Unfortunately, although not unexpectedly, only one RCT was found that compared MCH Handbook and its effect on maternal and child care and measured only one outcome important to decision making. Thus, due to the lack of RCTs and scarcity of outcomes, the search also included nonrandomized studies. Nonetheless, results from this study suggest that users of the MCH Handbook tended to have better outcomes of knowledge, practice, and attitude-related variables compared to non-users of the $\mathrm{MCH}$ Handbook. Further, narrative findings highlighted the $\mathrm{MCH}$ Handbook as a tool to increase ownership of immunization records, increase use of maternal health services, and increase knowledge related to topics such as exclusive breastfeeding. Thus, results from the meta-analysis and the narrative summary suggest that the MCH Handbook may have a positive effect on maternal child health and ultimately may be a useful tool to improve maternal and child health care and outcomes.

Similar results were found in a systematic review on the effect of the MCH Handbook. A systematic review conducted by Baequni and Nakamura (2012) [27] found that mothers who used the MCHHB during pregnancy had higher levels of knowledge (OR 1.44, 95\% CI: 1.22 -1.70) than whose did not use MCHHB during pregnancy. However, although the $\mathrm{MCH}$ Handbook may be a useful tool, evidence suggests varying uptake and utilization among various populations. One study found that utilization of the $\mathrm{MCH}$ Handbook is still less widespread than expected, especially among clients of private health services in Thailand [28]. A retrospective review by Nakamura (2010) [29] showed that 13,271 of guardians in Japan who visited 18-month health examinations of their children in 1999 used the MCH Handbook. As well, almost all guardians had read and written in their MCH Handbook, which shows that the MCH Handbook was highly utilized in Japan.

However, many guardians felt that the $\mathrm{MCH}$ Handbook was not so easy to utilize and the articles on dental health were not widely used. Thus, further research may be needed to examine the appropriateness of content and how the tool can be designed to ensure the tool is user-friendly. The results from this systematic review also align with the conclusions from the Tokyo Declaration, which noted that the $\mathrm{MCH}$ Handbook is critical to facilitate reciprocal communication between families and health care providers, and to empower women and their families to take an active role in their health care.

The MCH handbook may be an effective tool for communication with health providers and husbands, for both highly educated and less-educated women during their first pregnancy. Results suggested that although less-educated women rarely read the handbook themselves at home, they became familiar with health information and options related to $\mathrm{MCH}$ through personalized guidance that was provided by health providers at health facilities utilizing MCH handbook [30,31]. Research has also shown that women with lower education have received more of their health information from the MCH Handbook than women of other educational groups, which demonstrates that the $\mathrm{MCH}$ Handbook can be a beneficial health education tool even if a mother is not highly educated [29]. Thus, the MCH Handbook can be an effective tool to promote the maternal and child health care, and may offer an alternative tool to existing, fragmented home record tools for educating mothers for better maternal and child health care.

Similarly, Bhuiyan (2009) noted that the MCH handbook provides mothers and families with valuable information that can empower women to participate in their health care and actively engage with primary health care providers. The present review used a comprehensive and systematic search strategy. Rigorous procedures were used to screen potential papers, and quality of papers was thoroughly assessed using GRADE criteria. However, there are some notable limitations of this review. The quality of many of the studies was relatively low due to small sample sizes. Although restricting the search to only randomized controlled trials could have potentially provided the highest quality of evidence, there was a dearth of RCTs on this topic. Thus, the present search included nonrandomized controlled trials, which can be heavily influenced by confounders.

As can be seen in Figures 2-4, many studies were likely heavily influenced by selection bias, performance bias, and detection bias. Additionally, there was a broad range of variables reported in the studies included in the meta-analysis. The range of variables reported resulted in difficulty determining heterogeneity. Additional research from other countries where the $\mathrm{MCH}$ Handbook has been implemented to further discern the effect of the MCH Handbook in maternal and child health care at a global level, since results from a few selected countries may not be generalizable to all mothers around the world.

\section{References}

1. Murray CJ, Laakso T, Shibuya K, Hill K, Lopez AD (2007) Can we achieve millennium development goal 4 ? New analysis of country trends and forecasts of under-5 mortality to 2015. Lancet 370: 10401054.

2. Margaret $\mathrm{C}$ Hogan, Kyle J Foreman, Mohsen Naghavi, Stephanie $Y$ Ahn, Mengru Wang, et al. (2010) Maternal mortality for 181 countries, 1980-2008: A systematic analysis of progress towards millennium development goal 5. Lancet 375: 1609-1623.

3. Rajaratnam JK1, Marcus JR, Flaxman AD, Wang H, Levin-Rector A, et al. (2010) Neonatal, post neonatal, childhood, and under-5 mortality for 187 countries, 1970-2010: A systematic analysis of progress towards millennium development goal 4. Lancet 375: 1988-2008.

4. WHO, UNICEF, UNFPA, and The World Bank. (2010) Trends in maternal mortality: 1990 to 2008 . 
Citation: Bhuiyan S, Begum H, Deena, Ehsan S, Jamal Shah S, et al. (2017) Maternal and Child Health (MCH) Handbook and Its Effect on Maternal and Child Health Care: A Systematic Review and Meta-Analysis. J Community Med Public Health: CMPH-109.

5. Lawn JE, Cousens S,Zupan J (2005) Lancet neonatal survival steering team. 4 million neonatal deaths: When? Where? Why? Lancet 365: 891-900.

6. Ban, K.-M. (2010) United Nations secretary-general: Global strategy for women's and children's health,

7. Clark, A, Sanderson C (2009) Timing of children's vaccination in 45 low-income and middle-income countries: An analysis of survey data. Lancet 373: 1543-1549.

8. Kerber KJ, de Graft-Johnson JE, Bhutta ZA, Okong P, Starrs Aet al. (2007) Continuum of care for maternal, newborn and child health: From slogan to service delivery. Lancet 370: 1358-1369.

9. Bhuiyan SU and Nakamura Y (2009) Continuity of Maternal, Neonatal and Child Health Care through MCH Handbook for Ensuring the Quality of Life. $2008 \mathrm{MCH}$ handbook Conference Report.

10. Gertler PJ, Martinez S, Premand P, Rawlings LB, Vermeersch CMJ (2011). Impact Evaluation in Practice. The International Bank for Reconstruction and Development / The World Bank. 1818 H Street NW. Washington DC 20433. 1-266.

11. Homer CSE, Davis GK, Everitt LS (1999) The introduction of a woman held record into a hospital antenatal clinic: The bring your own records study. Australian and New Zealand Journal of Obstetrics and Gynaecology 39: 54-57.

12. Higgins JPT, Green S (2011) Cochrane Handbook for Systematic Reviews of Interventions.

13. Guyatt GH, Oxman AD, Vist GE, Kunz R, Falck-Ytter Y, et al (2008). GRADE: an emerging consensus on rating quality of evidence and strength of recommendations. BMJ 336: 924-926.

14. Aiga H, Nguyen VD, Nguyen CD, Nguyen TT, Nguyen LT (2006) Knowledge, attitude and practices: assessing maternal and child health care Handbook intervention in Vietnam. BMC public health 9: 16-129.

15. Bhuiyan SU, Nakamura Y, Qureshi NA (2006). Study on the Development and Assessment of Maternal and Child Health (MCH) Handbook in Bangladesh. Journal of Public Health and Development 4: 45-59.

16. Kawakatsu Y, Sugishita T, Oruenjo K, Wakhule S, Kibosia K, et al (2015) Effectiveness of and factors related to possession of a mother and child health Handbook: an analysis using propensity score matching. Health education research.30:935-946.

17. Mori R, Yonemoto N, Noma H, Ochirbat T, Barber E, et al. (2015) The Maternal and Child Health (MCH) Handbook in Mongolia: a clusterrandomized, controlled trial. PloS one 10: e0119772.

18. Osaki K, Hattori T, Kosen S (2013) The role of home-based records in the establishment of a continuum of care for mothers, newborns, and children in Indonesia. Global health action 6: 1-12.
19. Osaki K, Kosen S, Indriasih E, Pritasari K, Hattori T (2015) Factors affecting the utilisation of maternal, newborn, and child health services in Indonesia: the role of the Maternal and Child Health Handbook. Public health 129: 582-586.

20. Yanagisawa S, Soyano A, Igarashi H, Ura M, Nakamura Y (2015) Effect of a maternal and child health Handbook on maternal knowledge and behaviour: a community-based controlled trial in rural Cambodia. Health policy and planning 30: 1184-1192.

21. Dagvadorj A, Nakayama T, Inoue E, Sumya N, Mori R (2017) Cluster randomised controlled trial showed that maternal and child health Handbook was effective for child cognitive development in Mongolia. Acta paediatrica 106: 1360-1361.

22. Fujimoto S, Nakamura Y, Ikeda M, Takeda Y, Higurashi M (2001) Utilization of Maternal and Child Health Handbook in Japan. [Nihon koshu eisei zasshi] Japanese journal of public health 48: 486-494.

23. Hagiwara A, Ueyama M, Ramlawi A, Sawada Y (2013) Is the Maternal and Child Health $(\mathrm{MCH})$ Handbook effective in improving healthrelated behavior? Evidence from Palestine. Journal of public health policy 34: 31-45.

24. Jeong IS (2004) The Relationship between Retention of the Maternal Child Health Handbook, Awareness of DPT Additional Immunization and DPTAdditional Immunization. J Korean Community Nurs 15: 76-83.

25. Kusmayati A, Nakamura $Y$ (2007) Increased utilization of maternal health services by mothers using the maternal and child health handbook in Indonesia. J Int Health 22: 143-151.

26. Osaki K, Hattori T, Kosen S, Singgih B (2009) Investment in homebased maternal, newborn and child health records improves immunization coverage in Indonesia. Transactions of the Royal Society of Tropical Medicine and Hygiene 103: 846-848.

27. Baequni, Nakamura Y (2012) Is Maternal and Child Health Handbook effective? Meta-Analysis of the Effects of MCH Handbook. Journal of International Health 27: 121-127

28. Isarnurug $S$ (2009) Maternal and child health (MCH) Handbook in the world. Maternal and Child Health Handbook in Thailand. J of International Health 24: 61-66.

29. Nakamura Y (2010). Maternal and Child Health Handbook in Japan. JMAJ 53: 259-265.

30. Takeuchi J, Sakagami Y, Perez RC (2016) The Mother and Child Health Handbook in Japan as a Health Promotion Tool: An Overview of Its History, Contents, Use, Benefits, and Global Influence. Global pediatric health

31. Palombo CN, Duarte LS, Fujimori E, Toriyama AT (2014) Use and records of child health Handbook focused on growth and development. Revista da Escola de Enfermagem da US. 